\title{
Constraining Supersymmetry using the relic density and the Higgs boson
}

\section{Sophie Henrot-Versillé ${ }^{* \dagger}$}

LAL, CNRS/IN2P3, Orsay Cedex, France

E-mail: versile@lal.in2p3.fr

\begin{abstract}
I will present the impact of the recent measurements of Planck, LHC experiments and Xenon100 in terms of constraints on supersymmetric parameters. I will consider two models: mSUGRA for an illustration, and a more general Minimal Supersymmetric Standard Model with 13 free parameters. The analysis is done with the SFitter tool allowing a comparison of Bayesian and profile likelihoods results. I will pin point how the allowed structures in the parameter spaces are driven by the combination of the dark matter annihilation mechanisms and the light Higgs mass predictions.
\end{abstract}

XXII. International Workshop on Deep-Inelastic Scattering and Related Subjects,

28 April - 2 May 2014

Warsaw, Poland

\footnotetext{
* Speaker.

†in collaboration with Tilman Plehn, Michael Rauch, Dirk Zerwas, Stéphane Plaszczynski, Benjamin Rouillé d'Orfeuil, and Marta Spinelli
} 
The analysis, presented here, is a short view of Ref. [1]. It aims at studying the constraints that can be set on the SUSY parameters space using the most recent experimental constraints known today: mainly the Higgs mass, and the cold dark matter density in the Universe measured, respectively, at the LHC and by the Planck collaboration, as well as the Xenon100 results. We make use of the SFITTER toolkit [2]. Since the SUSY models are numerous and the parameter space very wide, we have restricted ourselves to the mSUGRA [3] model and a 13 parameters TeV scale MSSM.

Similar studies have been performed by other groups. For instance, FitTINo has studied the impact of LHC data and WMAP-7year results [4] on two models, mSUGRA and a non-universal Higgs model. The MASTERCODE group has performed a likelihood study of the same mSUGRA and non-universal Higgs models including Xenon100 results [5]. A specific analysis with Planck data, the Higgs mass measurement, and Xenon100 in the TeV-scale MSSM exists with a focus on light neutralino dark matter [6]. Results similar to ours have recently been published in Ref. [7] and by the BayesFITS group, including the study of a 9-parameter MSSM in Ref [8]. A non-exhaustive list of other, similar analyses is given in Ref. [9]. None are using up to date measurements in a parameter space as wide as the one presented here.

\section{Data and Context}

The measurements and the assumed theoretical uncertainties entering the analysis are summarized in table 1. The top mass [10] is a model parameter and a measurement. An additional dark matter related input is the upper limit on the elastic LSP-Nucleon cross section as function of the LSP mass from the analysis of the Xenon100 225 days $\times 34 \mathrm{~kg}$ dataset [16].

\begin{tabular}{l|cc}
\hline measurement & value and error & \\
\hline$m_{h}$ & $(126 \pm 0.4 \pm 0.4 \pm 3) \mathrm{GeV}$ & {$[11]$} \\
\hline$\Omega_{c d m} h^{2}$ Planck & $0.1187 \pm 0.0017 \pm 0.012$ & {$[12]$} \\
\hline $\mathrm{BR}\left(B_{s} \rightarrow \mu^{+} \mu^{-}\right)$ & $\left(3.2_{-1.2}^{+1.5} \pm 0.2\right) \times 10^{-9}$ & {$[13]$} \\
$\mathrm{BR}\left(b \rightarrow X_{s} \gamma\right)$ & $(3.55 \pm 0.24 \pm 0.09) \times 10^{-4}$ & {$[14]$} \\
$\Delta \mathrm{a}_{\mu}$ & $(287 \pm 63 \pm 49 \pm 20) \times 10^{-11}$ & {$[15]$} \\
$m_{t}$ & $(173.5 \pm 0.6 \pm 0.8) \mathrm{GeV}$ & {$[10]$} \\
\hline
\end{tabular}

Table 1: Some of the key measurements used in our analysis, including the error. The last number is the theoretical uncertainty on the supersymmetric prediction, except for the $\operatorname{BR}\left(b \rightarrow X_{s} \gamma\right)$ and $m_{t}$ for which no theoretical uncertainty is considered.

The prediction of the light MSSM Higgs mass is calculated with SUSPECT2 [17] while the Higgs branching ratios are computed using SUSY-HiT and HDECAY [18]. The supersymmetric contribution to the cold dark matter density is calculated with MicroMegas [19]. For the electroweak precision observables we rely on SUSYPoPE [20]. Finally, we use SUSPECT2 [17] and Micromegas [19] to compute the $B$ observables and $(g-2)_{\mu}$. SFitTER primarily relies on SUSPECT2 [17] for the renormalization group evolution and the computation of the supersymmetric mass spectrum. In addition, we use SoftSUSY [21] to test our results.

The analysis presented below proceeds in two steps. First, we construct a fully exclusive loglikelihood map in the model parameters using a set of Markov Chains with a Breit-Wigner proposal 

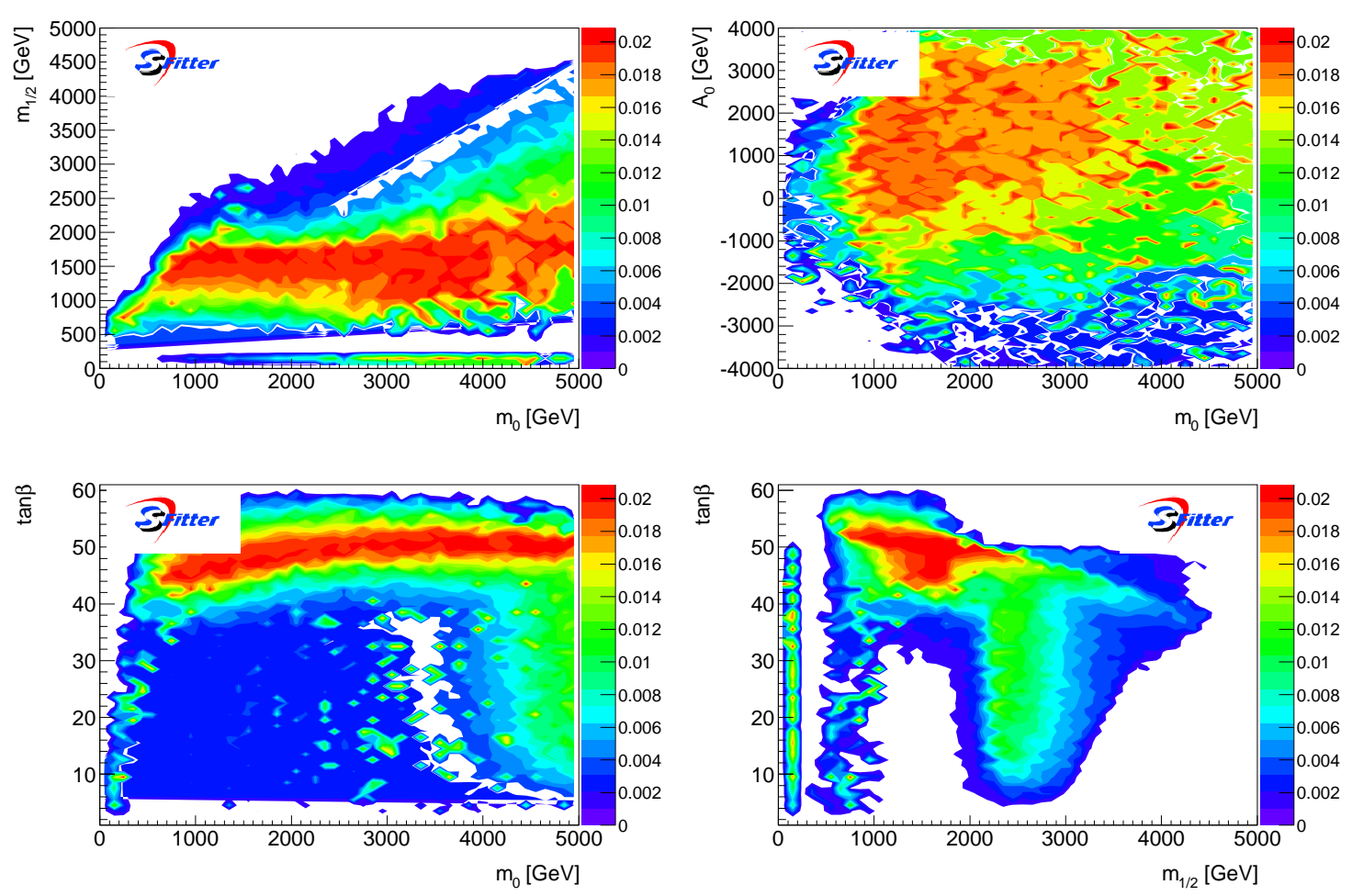

Figure 1: Profile likelihood projections onto the $\left(m_{0}, m_{1 / 2}\right)$ plane, the $\left(m_{0}, A_{0}\right)$ plane, the $\left(m_{0}, \tan \beta\right)$ plane, and the $\left(m_{1 / 2}, \tan \beta\right)$ plane. All results are based on the Planck measurement and assume $\mu>0$.

function. We then build $2 \mathrm{~d}$ parameters maps using profile likelihood (marginalization has also been performed but not presented here, more details can be found here [1]).

\section{2. mSUGRA analysis}

mSUGRA is described with four parameters at the GUT scale and a sign: $m_{0}, m_{1 / 2}, A_{0}, \tan \beta$ and $\operatorname{sign}(\mu)$. The light Higgs mass measurement constrains the parameter space through the stop sector. Our parameter space is bounded by $m_{0}<5 \mathrm{TeV}, m_{1 / 2}<5 \mathrm{TeV},\left|A_{0}\right|<4 \mathrm{TeV}$, and $\tan \beta<61$. The corresponding 2 dimensionnal profile likelihood contours are shown on figure 1 in the case of $\mu>0$.

The first observation is the absence of a clear preference in the $m_{0}$ values. In contrast, the dark matter relic density favors three distinct regions in $m_{1 / 2}$ :

1. the narrow stau co-annihilation strip with $m_{1 / 2}<1 \mathrm{TeV}$ and $m_{0}<500 \mathrm{GeV}$ at moderate $\tan \beta$. The mass of the lightest slepton $\tilde{\tau}_{1}$ is very close to the LSP mass.

2. the $A$-funnel region with $m_{1 / 2} \approx 1.7 \mathrm{TeV}$ and $\tan \beta \approx 50$, where the LSP mass around $745 \mathrm{GeV}$ is roughly half the heavy Higgs masses $m_{A, H}$ and the heavy Higgs states have a sizeable width to allow for a spread-out $s$-channel annihilation.

3. the $h$-funnel region with $m_{1 / 2} \approx 130 \mathrm{GeV}$, where the bino-LSP mass of $60 \mathrm{GeV}$ is about half the mass of the lightest Higgs. The dominant dark matter annihilation process is the resonant 
$s$-channel annihilation via the lightest Higgs boson. Because of the link between the LSP and gluino masses, this channel could typically be ruled out by direct LHC searches.

\section{MSSM analysis}

The most general MSSM contains a large number of parameters, of which we identify 17 which will affect current LHC and dark matter measurements [2]. Moreover, the absence of evidence for supersymmetric particles at the LHC leads us to effectively decouple some of the masses to values well about the $\mathrm{TeV}$ scale. In this analysis all squark mass parameters with the exception of the stop sector are fixed at $2 \mathrm{TeV}$. The same value is assumed for the gluino mass parameter $M_{3}$. This way gluinos and light-flavor squarks move outside the region excluded by the LHC. The trilinear mass parameter $A_{b}$ is assumed to be zero. The first-generation slepton parameters are identified with their second-generation counter parts. This leaves 13 supersymmetric parameters to be explored: $\tan \beta$, the electroweak gaugino mass parameters $\left(M_{1}, M_{2}\right)$, the smuon and stau sectors $\left(M_{\tilde{\mu}_{L, R}}, M_{\tilde{\tau}_{L, R}}, A_{\tau}\right)$, the stop sector $\left(M_{\tilde{q}_{3 L}}, M_{\tilde{\tau}_{R}}, A_{t}\right)$, the heavy Higgs mass $m_{A}$, and the higgsino mass parameter $\mu$. As for mSUGRA we also fit for the top mass. Our parameter space is bounded by $\tan \beta<61,\left(M_{1}, M_{2}\right)<4 \mathrm{TeV},\left(M_{\tilde{\mu}_{L / R}}, M_{\tilde{\tau}_{L / R}}, M_{\tilde{q}_{3 L}}, M_{\tilde{t}_{R}}\right)<5 \mathrm{TeV},\left(\left|A_{\tau}\right|,\left|A_{t}\right|\right)<4 \mathrm{TeV}, m_{A}<5 \mathrm{TeV}$ and $|\mu|<2 \mathrm{TeV}$.

The measured light Higgs mass essentially depends on three parameters: the heavy Higgs mass scale $m_{A}$, which has to be large to accommodate the $126 \mathrm{GeV}$ measurement; $\tan \beta$ which has to be large enough to not delay the decoupling regime in $m_{A}$; and finally the geometric mean of the two stop masses $\sqrt{m_{\tilde{t}_{1}} m_{\tilde{t}_{2}}}$, which again has to be large. In terms of MSSM parameters the latter needs to be computed from the three entries in the stop mass matrix, including $A_{t}$. The stop masses are the key parameters, but are neither strongly related to the dark matter sector nor to the light-flavor squark-gluino mass plane. In addition, they are directly linked to the solution of the hierarchy problem and hence to the motivation of supersymmetry.

In Figure 2 we show the profile likelihoods in the neutralino and chargino sector $M_{1}, M_{2}$, and $\mu$. Those maps favor five regions, three of which directly correspond to the mSUGRA case:
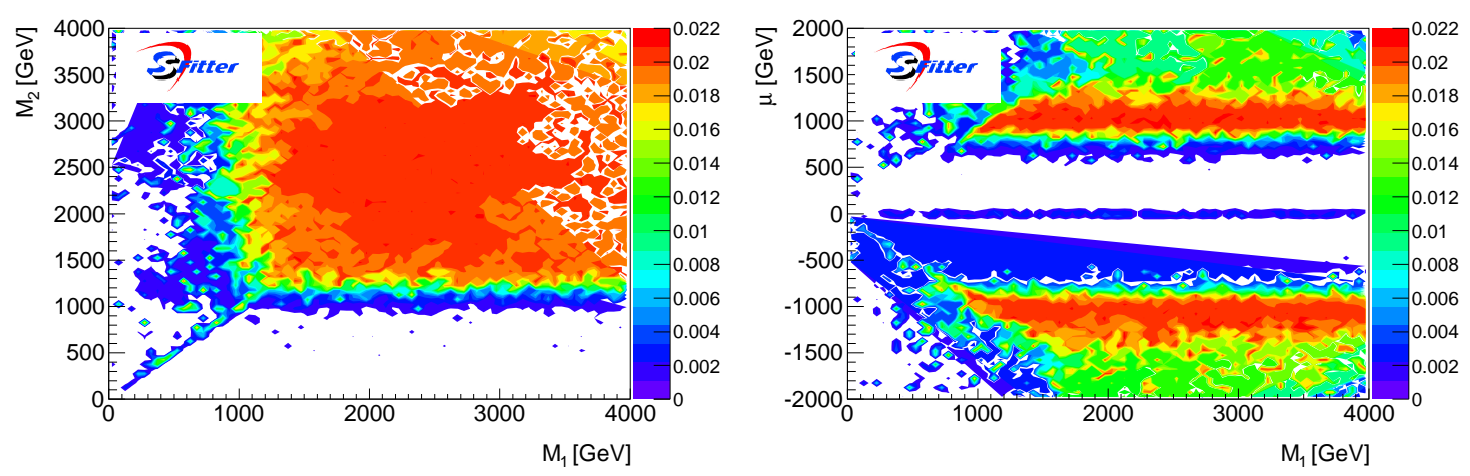

Figure 2: Profile likelihood projection onto the $\left(M_{1}, M_{2}\right)$ plane (left) and the $\left(M_{1}, \mu\right)$ plane (right) for the Planck measurements. 
1. the stau co-annihilation strip diagonal in $M_{1}$ vs $M_{2}$ at relatively small values. Here, the mass of the lightest slepton $\tilde{\tau}_{1}$ is very close to the LSP mass.

2. the A-funnel region where the LSP mass is about half the heavy Higgs mass. This MSSM region behaves the same way as discussed for the simpler mSUGRA model. In Figure 2 it contributes to the bulk region of the $M_{1}$ vs $M_{2}$ plane as well as to the correlated patterns in the $M_{1}$ vs $\mu$ plane.

3. the $h$-funnel region at low $M_{1} \sim 63 \mathrm{GeV}$ almost independent of $M_{2}$. Unlike for mSUGRA the gluino mass is now an independent parameter, so the direct LHC searches decouple from the dark matter sector. Because the corresponding MSSM parameter space is tiny, the funnel appears only as distinct sets of points in Figure 2. We have checked that it actually is a narrow line.

4. a bino-higgsino region which appears as a strip in the $M_{1}$ vs $\mu$ plane for $\mu<0$ and $\left|M_{1}\right| \approx|\mu|$. The dark matter annihilation proceeds through different neutral and charged Higgs-mediated channels, including chargino co-annihilation and dominantly third-generation quarks in the final state. The latter includes the $b \bar{b}$ final state from the $A$-funnel.

5. a large higgsino region with $M_{1}, M_{2}>1.2 \mathrm{TeV}$, split in two almost symmetric solutions $\mu \approx \pm 1.2 \mathrm{TeV}$. Chargino co-annihilation dominates the prediction of the relic density with first and second generation quarks in the final state.

\section{Conclusion}

The allowed regions of supersymmetric parameter space can best be categorized by the dark matter annihilation channel. In mSUGRA we found two valid regions, a narrow stau co-annihilation region at moderate $\tan \beta$ and a large $A$-funnel region. Stop co-annihilation survives the light Higgs mass constraint, but resides outside our tested range of model parameter space, while the focuspoint region seems to be ruled out.

In the TeV-scale MSSM we found narrow allowed regions corresponding to stau co-annihilations and the light-Higgs funnel annihilation. The heavy Higgs funnel becomes part of a large parameter region where the lightest neutralino is a mixed bino-higgsino state, annihilating to thirdgeneration fermions. Chargino co-annihilation occurs with a charged Higgs funnel. In addition, we observed a large higgsino region with chargino and neutralino co-annihilation through gauge boson and into light-flavor quarks. Finally, stop co-annihilation again resides outside our range of model parameters.

In terms of the supersymmetric Lagrangian we found that the positive measurements like the relic density or the Higgs mass generally push supersymmetry toward a high new physics mass scale. The absence of signals for new physics at the $8 \mathrm{TeV}$ run of the LHC puts little tension into the parameter analysis. Nevertheless, several of the parameter regions corresponding to different dark matter annihilation can be probed by the LHC running at $13 \mathrm{TeV}$. 


\section{References}

[1] S. Henrot-Versillé, R. Lafaye, T. Plehn, M. Rauch, D. Zerwas, S. Plaszczynski, B. Rouillé d'Orfeuil, M. Spinelli, Phys. Rev. D 89, 055017 (2014).

[2] R. Lafaye, T. Plehn and D. Zerwas, arXiv:hep-ph/0404282; R. Lafaye, T. Plehn, M. Rauch and D. Zerwas, Eur. Phys. J. C 54, 617 (2008).

[3] L. E. Ibañez, Phys. Lett. B 118, 73 (1982); J. R. Ellis, D. V. Nanopoulos and K. Tamvakis, Phys. Lett. B 121, 123 (1983); L. Alvarez-Gaumé, J. Polchinski and M. B. Wise, Nucl. Phys. B 221, 495 (1983); K. Inoue, A. Kakuto, H. Komatsu and S. Takeshita, Prog. Theor. Phys. 68, 927 (1982) [Erratum-ibid. 70, 330 (1983)]; A. H. Chamseddine, R. Arnowitt and P. Nath, Phys. Rev. Lett. 49, 970 (1982).

[4] P. Bechtle, K. Desch and P. Wienemann, arXiv:hep-ph/0412012; P. Bechtle, T. Bringmann, K. Desch, H. Dreiner, M. Hamer, C. Hensel, M. Krämer and N. Nguyen et al., JHEP 1206 (2012) 098.

[5] O. Buchmüller, R. Cavanaugh, M. Citron, A. De Roeck, M. J. Dolan, J. R. Ellis, H. Flacher and S. Heinemeyer et al., Data” Eur. Phys. J. C 72, 2243 (2012).

[6] C. Boehm, P. S. B. Dev, A. Mazumdar and E. Pukartas, JHEP 1306, 113 (2013).

[7] T. Cohen and J. G. Wacker, arXiv:1305.2914 [hep-ph].

[8] A. Fowlie, M. Kazana, K. Kowalska, S. Munir, L. Roszkowski, E. M. Sessolo, S. Trojanowski and Y. -L. S. Tsai, Phys. Rev. D 86, 075010 (2012); A. Fowlie, K. Kowalska, L. Roszkowski, E. M. Sessolo and Y. -L. S. Tsai, Phys. Rev. D 88, 055012 (2013).

[9] See e.g. L. Roszkowski, E. M. Sessolo and Y. -L. S. Tsai, Phys. Rev. D 86, 095005 (2012); C. Strege, G. Bertone, F. Feroz, M. Fornasa, R. Ruiz de Austri and R. Trotta, JCAP 1304, 013 (2013); B. Bhattacherjee, M. Chakraborti, A. Chakraborty, U. Chattopadhyay, D. Das and D. K. Ghosh, arXiv:1305.4020 [hep-ph]; M. Cahill-Rowley, J. Hewett, A. Ismail and T. Rizzo, arXiv:1308.0297 [hep-ph].

[10] Particle Data Group: http://pdg.lbl.gov/2012/reviews/rpp2012-rev-standard-model.pdf

[11] ATLAS Collaboration, Science 338, 1576 (2012).

[12] P. A. R. Ade et al. [Planck Collaboration], arXiv:1303.5076 [astro-ph.CO].

[13] R. Aaij et al. [LHCb Collaboration], Phys. Rev. Lett. 110, 021801 (2013).

[14] Particle Data Group http://pdg.lbl.gov/2012/reviews/rpp2012-rev-b-meson-prod-decay.pdf

[15] Particle Data Group http://pdg.lbl.gov/2012/reviews/rpp2012-rev-g-2-muon-anom-mag-moment.pdf

[16] E. Aprile et al. [XENON100 Collaboration], Phys. Rev. Lett. 107, 131302 (2011); E. Aprile et al. [XENON100 Collaboration], Phys. Rev. Lett. 109, 181301 (2012).

[17] A. Djouadi, J. -L. Kneur and G. Moultaka, Comput. Phys. Commun. 176, 426 (2007).

[18] A. Djouadi, J. Kalinowski and M. Spira, Comput. Phys. Commun. 108, 56 (1998); M. Mühlleitner, A. Djouadi and Y. Mambrini, arXiv:hep-ph/0311167; A. Djouadi, M. M. Mühlleitner and M. Spira, Acta Phys. Polon. B 38, 635 (2007).

[19] G. Belanger, F. Boudjema, P. Brun, A. Pukhov, S. Rosier-Lees, P. Salati and A. Semenov, Comput. Phys. Commun. 182, 842 (2011).

[20] S. Heinemeyer, W. Hollik, A. M. Weber and G. Weiglein, JHEP 0804, 039 (2008); A. Weber, private communication.

[21] B. C. Allanach, Comput. Phys. Commun. 143, 305 (2002). 